\title{
Specific features of defect and mass transport in concentrated fcc alloys
}

\author{
Yuri N. Osetsky ${ }^{1}$, Laurent K. Béland and Roger E. Stoller \\ Materials Science and Technology Division, Oak Ridge National Laboratory \\ Oak Ridge, Tennessee 37831-6138, USA
}

Submitted: December 11, 2015

Modified: April 14, 2016

Copyright notice: This manuscript has been authored by UT-Battelle, LLC under Contract No. DE-AC05-00OR22725 with the U.S. Department of Energy. The United States Government retains and the publisher, by accepting the article for publication, acknowledges that the United States Government retains a non-exclusive, paid-up, irrevocable, world-wide license to publish or reproduce the published form of this manuscript, or allow others to do so, for United States Government purposes. The Department of Energy will provide public access to these results of federally sponsored research in accordance with the DOE Public Access Plan (http://energy.gov/downloads/doe-public-access-plan).

${ }^{1}$ Corresponding author e-mail: osetskiyyn@ornl.gov 


\begin{abstract}
Diffusion and mass transport are basic properties that control materials performance, such as phase stability, solute decomposition and radiation tolerance. While understanding diffusion in dilute alloys is a mature field, concentrated alloys are much less studied. Here, atomic-scale diffusion and mass transport via vacancies and interstitial atoms are compared in fcc $\mathrm{Ni}$, Fe and equiatomic Ni-Fe alloy. High temperature properties were determined using conventional molecular dynamics on the microsecond timescale, whereas the kinetic activation-relaxation (k-ART) approach was applied at low temperatures. The k-ART was also used to calculate transition states in the alloy and defect transport coefficients. The calculations reveal several specific features. For example, vacancy and interstitial defects migrate via different alloy components, diffusion is more sluggish in the alloy and, notably, mass transport in the concentrated alloy cannot be predicted on the basis of diffusion in its pure metal counterparts. The percolation threshold for the defect diffusion in the alloy is discussed and it is suggested that this phenomenon depends on the properties and diffusion mechanisms of specific defects.
\end{abstract}

\title{
1. Introduction
}

Structural materials, other than intermetallics, are typically based on one main alloying element (solvent) with others added in lower proportions (solutes) to improve desired properties. A new class of alloys, referred to as high-entropy alloys (HEA), was recently proposed [1]. The main feature of these alloys is the near-equiatomic ratio of its components. These new materials are under intensive study. Important questions related to their structural stability, including phase stability, precipitation and segregation under thermal or/and irradiation conditions, are under investigation. The main atomistic mechanism for microstructural evolution is diffusion via lattice defects. Under thermal and irradiation conditions these correspond to vacancies, interstitial atoms and small clusters of each. General 
considerations based on possible fluctuations of local potential energy suggest that diffusion in HEAs can be slower than in pure components (sluggish diffusion effect) [1,2]. A few experimental studies [3,4] have confirmed this qualitatively in $\mathrm{Co}-\mathrm{Cr}-\mathrm{Fe}-\mathrm{Mn}-\mathrm{Ni}$. However, the details of defect diffusion mechanisms in such concentrated alloys are not well understood and this question should be resolved in order to create models with predictive capabilities.

Diffusion theory is well developed for dilute alloys, where the process can be decomposed into a limited number of elementary processes, i.e. defect jumps of a particular type. Knowing defect energies and migration barriers around an isolated impurity, the diffusion process can be described theoretically using the classical theories by Darken [5] or Manning [6] and their developments, such as analytical multifrequency models [7,8], a statistical treatment of local configurations using flexible self-consistent mean field theory [9], or the master equation [10]. Alternatively, one can use Monte Carlo (MC) methods to model the defect path through the alloy [11-14]. These approaches have two main common issues. First, a number of effective parameters must be determined to describe the kinetics of the processes. These include local defect binding energies, migration barriers and frequencies for all types of jumps in the system. These must be calculated in advance using empirical interatomic potentials, $a b$ initio techniques or estimated on-the-fly as in lattice kinetic MC modeling. One should note that in addition to potential energy contributions, other factors are involved in the diffusion process; these include electrostatic, vibrational entropy, magnetism [15]. Second, statistical approximations must be made to average the probabilities of visiting different defect configurations and executing jumps in order to use the abovementioned techniques [5-14]. All these methods work well in non-concentrated alloys, where the number of unique configurations is small and/or local properties can be estimated as perturbations of the matrix. Important challenges appear in concentrated alloys where the number of nonequivalent configurations, local energies, barriers and frequencies for atomic displacements/jumps is too large to be predicted $a$ 
priori. Also, the vibrational contributions to the entropy in concentrated alloy are more complex.

The most accurate approach so far was based on ab initio MD [16]. The authors were able to simulate self-interstitial diffusion in pure $\mathrm{Ni}$ and a $\mathrm{Ni}-18$ at.\% $\mathrm{Cr}$ alloy. Interstitial tracer diffusivities obtained for pure Ni and in the alloy were transferred to a rate theory model to predict radiation-induced segregation. The model predicted that both vacancy and interstitial diffusion play a role in grain boundary segregation. Unfortunately, the length scales and timescales required to accumulate sufficient diffusion statistics are beyond the reach of DFT-based MD. To perform a detailed study of the kinetics that drives diffusion, one has to rely on conventional MD. Attempts to apply MD to diffusion in random alloys [1723] have been primarily focused on dilute alloys and the interactions between defects and solutes.

In addition, diffusion and mass transport in concentrated alloys above the percolation threshold might have specific features that have not yet been studied directly under dynamic conditions. Indeed, the site percolation threshold concentration in a binary fcc alloy is about 20 at $\%$ [24]. Previous studies have not explored concentrations above this threshold, where each of the two alloying components has a continuous link with atoms of its own kind. In regards to diffusion processes, this means that point defects can continuously move within a given subsystem. Equiatomic alloys are particularly interesting, since none of the components have preference in percolation sequences. These issues are considered in the present research where diffusion of vacancies and interstitial atoms in pure fcc $\mathrm{Ni}$ and $\mathrm{Fe}$, and an equiatomic NiFe alloy is studied. In this paper, only a limited range of diffusion data is reported because the main aim is not to report the diffusion mechanisms in details, but rather to demonstrate that concentrated alloys exhibit specific diffusion and mass transport features. For this reason, our study mainly focused on tracer diffusion, which is simply defined by atomic displacements. To understand other alloying effects, the defect jump correlations and transport coefficients were also analyzed. 


\section{Modeling approaches}

Several atomic-scale models were used. A brief description of the models, data treatment, validity and accuracy is presented in the Appendix. The present work treats mainly tracer diffusion coefficients, $D^{*}$, because they are the most reliable output of MD which do not depend on trajectory decomposition parameters or defect characterization techniques. The present MD study is focused on producing results of maximum accuracy by reaching times on the order of a $\mu \mathrm{s}$. An example of atomic squared displacements (ASDs) for vacancy and interstitial diffusion is presented in Fig.1. In this example all the ASDs are approximated very well by linear functions of time that define tracer diffusion coefficients with a high statistical accuracy. The error bars are not presented for $D^{*}$ because they are smaller than the symbols. The ASDs presented in Fig.1 for the alloy were produced by $\sim 2 \times 10^{5}$ and $\sim 4 \times 10^{3}$ jumps of an interstitial atom and vacancy correspondingly. The insets in Fig.1 show the tracer diffusion coefficients of the components extracted from ASDs. Diffusivities in $\mathrm{Ni}$ and Fe are significantly different underlying the interstitial in the alloy mainly exchanges with $\mathrm{Ni}$ atoms, while the vacancy mainly displaces Fe atoms. Vacancies in $\mathrm{Ni}$ and in the alloy have quite high migration energies and a very low diffusivity at low temperature. Hence, even $\mu$ s-scale MD generates inaccurate statistics at temperatures below 1000K. For example only 145 and 352 vacancy jumps were observed in $\mathrm{Ni}$ and the alloy, respectively, during $\sim 1 \mu \mathrm{s}$ simulation at 900K, (see Fig. A1). In such cases, the kinetic activation relaxation technique (k-ART) [25] was applied, as described in the Appendix. It is very efficient at capturing vacancy defect diffusion in cases where MD fails due to a low defect jumps frequency.

Interatomic potentials (IAPs) determine all the properties of the system modeled by both MD and kART and selecting the right potential is an important issue. Three potential sets were considered for describing Ni-Fe [26-28]. The IAPs from [27] (B2011) and [28] (B2013) are fitted to a wide range of 
pure metal and alloy properties. Properties of vacancy and interstitial atoms in fcc Ni and Fe calculated with these potentials are presented in Table. 1 together with $a b$ initio data either used as target for the IAP fitting $[27,28]$ or calculated independently (such as the interstitial atom migration barrier in Ni [15]). Using both potential sets, vacancy and interstitial atom formation and migration energies were computed in the alloy using static techniques. Vacancy and interstitial diffusion in pure $\mathrm{Ni}, \mathrm{Fe}$, and the Ni-Fe alloy were computed using MD. Defect formation energies in pure metals and the distribution of their values in the alloy were expected to be important for defect dynamics. Comparisons with ab initio data [29] suggest that the B2011 [27] potential set generates the most physically accurate distributions. We therefore selected it for use in the study of defect dynamics in the concentrated NiFe alloy.

\section{Results.}

\subsection{Defects formation and transition states energy.}

Vacancy and interstitial atom formation energies in the NiFe alloy were studied. The computational details are described in the Appendix and the results are presented in Fig.2. The spectrum of formation energies is much narrower for vacancies than it is for interstitial atoms. The most energetically favorable interstitial defects have Ni-Ni composition, followed by mixed dumbbells, and Fe-Fe dumbbells. There is little overlap of the energy distributions for dumbbells of different composition, while the distributions for $\mathrm{Ni}$ and Fe vacancies almost fully overlap. Ni-vacancies are slightly energetically favored, with the a small energy difference between the maximum of the $\mathrm{Ni}$ and Fe vacancy distributions, $\sim 0.1 \mathrm{eV}$. In contrast, the difference between the maximum $\mathrm{Ni}-\mathrm{Ni}$ and $\mathrm{Ni}-\mathrm{Fe}$ dumbbell distributions is $\sim 0.5 \mathrm{eV}$. It can be assumed there is a clear preference for interstitials to maintain a Ni-Ni composition and a weak preference for vacant Ni sites. It should be noted that the chemical composition of an interstitial dumbbell can be 
unambiguously be determined, while the chemical composition of a vacancy is ill-defined.

Using k-ART the transition states that were crossed during more than 3000 vacancy or interstitial jumps in the Ni-Fe alloy were analyzed at different temperatures. The activation energies for transition states at $900 \mathrm{~K}$ for the vacancy and $600 \mathrm{~K}$ for the interstitial atom are presented in Fig.3. The local chemical features of these states were not analyzed in detail, but there is a large number of chemically non-equivalent and structurally similar states that have different activation energies. The spectra presented in Fig. 3 show that activation energy distributions for interstitial atoms and vacancies in the alloy are quite similar with a variation about $\pm 0.25-0.30 \mathrm{eV}$ around the mean values which are $0.28 \mathrm{eV}$ for interstitial jumps and $0.97 \mathrm{eV}$ for vacancy jumps. Note that the maximum of the interstitial's activation energy distribution is very close to the minimum the vacancy's but that there is no significant overlap. It is also interesting to note that there are a number of very low activation energy transition states for the both vacancies and interstitials. These states are usually related to some metastable configuration along the defect migration path. The number of such states significantly increases in concentrated alloys making k-ART and any other on-the-fly kMC modeling quite expensive because of their frequent appearance (due to a low activation energy) but low efficiency in producing atomic displacements.

\subsection{Point defects diffusion.}

The tracer diffusion coefficients obtained for interstitial atom and vacancy diffusion simulated in pure metals and the alloy by MD and k-ART are presented in Fig.4. The insets provide information on the effective activation energies deduced from the corresponding Arrhenius plots and a ratio of partial tracer diffusion coefficients for the alloy components, $D^{*}{ }_{N i} / D^{*}$ Fe

Several interesting features can be observed. In general, point defects are more mobile in fcc-Fe than in Ni. Activation energy, $E^{a c t}$, for defects diffusion in Ni obtained from MD modeling and their 
corresponding static barriers are similar. For the vacancy the MD activation energy is $1.32 \mathrm{eV}$ and the static barrier $E^{m}=1.17 \mathrm{eV}$; for the interstitial atom $E^{a c t}=0.32 \mathrm{eV}$ and $E^{m}=0.33 \mathrm{eV}$. However, the difference is quite significant for Fe. For the vacancy $E^{a c t}=0.92 \mathrm{eV}$ and $E^{m}=0.61 \mathrm{eV}$ while for the interstitial atom the MD energy is $0.09 \mathrm{eV}$ with a static barrier of $0.33 \mathrm{eV}$ (see Table.1 and Fig.4). Note that the activation energy of interstitial atom diffusion extracted from MD is lower than the static barrier whereas for vacancy this is opposite : $E^{a c t}>E^{m}$.

Another feature observed in Fig.4 is the difference in behavior between the vacancy and the interstitial atom in pure metals and the alloy. Indeed, the diffusivity of interstitial atoms in the alloy is much lower than in the pure metals. Moreover, the interstitial atom displaces mainly Ni atoms while moving through the alloy (see Fig. 1 as an example of ASDs at 700K). The ratio of partial diffusion coefficients of $\mathrm{Ni}$ and $\mathrm{Fe}, D_{N i} / D_{\mathrm{Fe}}$, in the alloy changes from $\sim 45$ at $600 \mathrm{~K}$ down to $\sim 10$ at $1100 \mathrm{~K}$. k-ART produces higher tracer diffusion coefficients in the alloy and $D_{N i} / D_{F e}$ ratios. As discussed in the Appendix, the current version of k-ART uses a constant attempt frequency for all transition states. This brings some inaccuracy to the value in the pre-exponential term of the diffusion coefficient. However, $E^{a c t}$ for interstitial atom diffusion from k-ART is rather close to that from MD, $0.39 \mathrm{eV}$ vs $0.40 \mathrm{eV}$ respectively. The vacancy moves through the alloy primarily through Fe sites (see ASDs at $1200 \mathrm{~K}$ in Fig.1 as an example). Its diffusivity in the alloy is much lower than in pure Fe and is very close to that in pure Ni. The activation energy for vacancy diffusion in the alloy, $1.14 \mathrm{eV}$, lies midway between the values for pure $\mathrm{Fe}, 0.95 \mathrm{eV}$, and $\mathrm{Ni}, 1.29 \mathrm{eV}$. As in the case of interstitial atoms, k-ART produces a slightly higher vacancy diffusivity than that obtained by MD, but a similar activation energy of $1.09 \mathrm{eV}$.

There are several different activation energies for defect diffusion that can be estimated by atomic scale modeling; these include the static barriers along the expected defect path, activation energies estimated through an Arrhenius-type treatment of defect jumps, defect diffusivities, and atomic squared 
displacements. Differences among these are due to the contributions of different types of defect jumps and the level of correlations included in the consideration. Among these, the static barriers, $E^{m}$, and activation energy for tracer diffusion estimated from the ASDs, $E^{a c t}$, are the two extremes. The former is just a set of possible defect trajectories that can be activated in the alloy such as the barriers that were activated during k-ART modeling of defect diffusion presented in Fig.3. The latter is effective activation energy for atomic displacements via the different transitions executed during the simulation. Each of these transitions has its own barrier and attempt frequency, as well as some correlation with the previous and subsequent transition states. These are exactly the energies obtained by MD and presented in insets in Fig.4. One can see that a simple average over the spectrum of all activated barriers (Fig.3) results in activation energies that are quite far from those estimated from tracer diffusivities (Fig.4). The explanation for this phenomenon is discussed in Section 4.

\subsection{Chemical anisotropy of defect diffusion.}

In the equiatomic alloy considered here, the interstitial atom and the vacancy choose their own chemically homogenous channel for diffusion. The interstitial atom diffuses mainly along the $\mathrm{Ni}$ channels. We have analyzed the composition of $<100>$ dumbbell configurations during its diffusion. The vast majority of the jumps were executed via $\mathrm{Ni}-\mathrm{Ni}$ dumbbells and only a few Ni-Fe jumps were detected at high temperatures. This is consistent with the temperature dependence of partial diffusion coefficients presented in the inset of Fig.4 for interstitial diffusion in the alloy where $D_{N i} / D_{F e}$ drops from $\sim 45$ at $600 \mathrm{~K}$ to $\sim 10$ at $1100 \mathrm{~K}$. In the case of vacancy diffusion, we analyzed the type of atom that jumps into the vacancy; the majority of them were identified as Fe. However, the MD modeling of vacancy diffusion was not accurate enough at low temperature, particularly at 900 and $1000 \mathrm{~K}$ (see e.g. Fig. A1). The $D_{F e} /$ $D_{N i}$ ratio presented in Fig.4 is clearly underestimated at low temperature but tends to increase with 
increasing simulation time. Although k-ART, overestimates values for the tracer diffusion coefficient and the $D_{\mathrm{Fe}} / D_{\mathrm{Ni}}$ ratio, it nevertheless predicts a qualitatively plausible $D_{\mathrm{Fe}} / D_{\mathrm{Ni}}$ ratio for all temperatures being studied.

The MD and k-ART modeling presented here unambiguously described chemical separation of mass flows during interstitial atom and vacancy diffusion. The equiatomic alloy provides the maximum possible venue for such separation because it consists of contiguous three-dimensional regions containing atoms of the same type. Therefore both the interstitial atom and the vacancy can migrate continuously in their preferred subsystem; this is exactly what happens at low temperature. At high temperature, both defects occasionally touch the other subsystem, as the decrease in chemical anisotropy of partial diffusion coefficients reflects. However, even at low temperature, when each defect migrates within a particular "chemical channel", the diffusion coefficient is significantly lower than that in the corresponding pure metal. This can be seen in Fig. 4 by comparing $D^{*}$ for interstitial diffusion in Ni and the alloy, and that of the vacancy in Fe and the alloy. There may be a number of reasons for this observation that defect properties in a given material embedded into a volume of another material are different from those in the pure material. We have investigated a particular reason for the spatial anisotropy that appears in highly concentrated alloys. In pure $\mathrm{Ni}$ and $\mathrm{Fe}$, the interstitial atom and the vacancy can jump to any of 12 closest lattice sites along $<110>$ directions. In the alloy, these directions are limited by the type of atom in the nearest sites, i.e. an interstitial atom prefers jumping to Ni sites, whereas a vacancy prefers Fe sites. This limits the number of directions in which a defect will jump and creates new correlations. Correlations in interstitial atom and vacancy jumps in pure metals and the alloy are presented in Fig.5. The correlation factor for vacancy jumps is similar in $\mathrm{Ni}$ and $\mathrm{Fe}$, and decreases from $\sim 0.9$ at low temperature to $\sim 0.8$ at high temperature. The temperature dependence of the correlation factor is different in the alloy: it is increases with temperature from $\sim 0.7$ to $\sim 0.8$. Similar but more significant changes can be observed for 
interstitial atoms. In pure metals the correlation factor slightly decreases from $\sim 0.5$ at low temperature to $\sim 0.4$ at high temperature whereas in the alloy it increases from $\sim 0.2$ to $\sim 0.4$ over the same temperature range. The lower correlation factor in the alloy contributes to a total decrease of tracer diffusion coefficients and this effect is stronger for the interstitial atom and lower temperatures.

The observed chemical anisotropy in defect diffusion causes a separation of flows of $\mathrm{Ni}$ and Fe and should contribute to alloy component segregation when diffusion occurs. To understand mass transport due to diffusion of different defects, transport coefficients, $L_{i j}$, $[8,30]$ were calculated for both vacancy and interstitial diffusion (see Appendix for definition). The effects are stronger at low temperatures therefore k-ART was used since MD is not efficient at these conditions. The value of transport coefficients was estimated applying trajectory decomposition similar to that used for defect diffusivities $[31,32]$. As for diffusion coefficients, the accuracy of transport coefficient estimates depends on the length of simulated trajectories. The convergence of $L_{i j}$ values versus the length of trajectory decomposition segments is shown in Fig.6 for interstitial diffusion at $600 \mathrm{~K}$ and vacancy diffusion at $900 \mathrm{~K}$. It can be seen that $\sim 3000$ jumps simulated by k-ART provide quite satisfactory convergence for decomposition segments longer than $\sim 40$ jumps. The calculated transport coefficients clearly demonstrate that the correspondence in direction of component and defect flows. $L_{I N T, F e}$ is equal to zero, which means that there is no Fe transport by interstitial atoms at this temperature. $L_{I N T, N i} \approx 3 \times 10^{-8} \mathrm{~m}^{2} / \mathrm{s}$, indicating that the interstitial atom provides quite efficient flow of $\mathrm{Ni}$ atoms in the same direction. The situation with vacancy diffusion is the opposite. $L_{V A C, N i}$ is equal to zero, indicating no transport of Ni atoms by vacancy diffusion, while $L_{V A C, F e} \approx-5 \times 10^{-11} \mathrm{~m}^{2} / \mathrm{s}$, indicating a relatively slow transport of $\mathrm{Fe}$ atoms in the direction opposite to vacancy diffusion.

\section{Discussion and conclusions.}


The atomic-level diffusion research presented here has a number of unique features. First, the maximum possible concentration for a binary alloy was investigated. Second, we presented the longest conventional MD diffusion simulations reported so far for such problems; the total modeling time for all the defects over all the temperatures studied, i.e. 31 individual simulations, exceeds $12 \mu$ s. Third, we complemented MD simulations with the mesoscale k-ART model at low temperatures where MD is much less efficient. In the majority of our simulations, only one alloy sample was considered. One may wonder if averaging over many different samples would lead to different results. To check this we have performed three additional kART simulations of vacancy diffusion at $1100 \mathrm{~K}$, each longer than 1 microsecond in time. The values of tracer diffusion coefficient are found to be within the range $(3.9-4.1) \times 10^{-11} \mathrm{~m}^{2} / \mathrm{s}$. We accepted this small difference in results as an evidence that even in the case of vacancy diffusion the trajectory affordable for modeling is long enough to represent properties of the alloy.

In addition to diffusion coefficients, the distribution of point defect formation and transition state energies in the equatomic alloy were calculated. These energies have a relatively wide spectrum which is an important feature of concentrated alloys. The formation energies define the subsystem in which interstitial atoms evolve and the energy of transition states defines the mobility of defects. These properties are usually used as inputs for transition state theory models. The accuracy of this approach is however an open question, as we illustrated with some examples. Even in the simple case of point defect diffusion in pure metals, there is a difference between the statically estimated energy barrier and tracer diffusion activation energy. Examples are presented in Table 1 for the two sets of Ni and Fe potentials. The general trend is that dynamically determined activation energies for vacancy migration are larger than static barriers, and with the opposite change for interstitial atoms. This conclusion is likely to be not universal for it depends on interatomic interactions and the vibrational properties of the material. Dynamic modeling within DFT could help to clarify this question. It is worth highlighting that deviations from 
Arrhenius behavior is within the statistical accuracy of our simulations, which makes the difference between the barriers extracted from MD and static calculations even more surprising. Resolution of this problem could not be obtained in the current investigation, but warrants further research.

A more complex question pertains to the prediction of defect diffusion. Given a spectrum of activation energies, what is the most appropriate method to obtain a relevant average value? The approaches used in dilute alloys where the number of transition states is countable (e.g. kMC) cannot be applied directly to concentrated alloys. Here, the k-ART serves this purpose. This extension of adaptive kMC samples a large number of possible events on-the-fly for each configuration encountered during diffusion. The total number of transition states is huge: each stable defect position is connected to tens, or even hundreds, of other stable and metastable positions. The spectra of transition states realized in k-ART simulations of an interstitial atom at $600 \mathrm{~K}$ and a vacancy at $1200 \mathrm{~K}$ are presented in Fig. 3. The simple average over the corresponding spectrum is $0.28 \mathrm{eV}$ for the interstitial atom and $0.97 \mathrm{eV}$ for the vacancy. However these energies are not equivalent to the effective migration energy of the corresponding diffusion process, $E^{m}$, since each energy should contribute with its own waiting time that depends on the energy value and attempt frequency for the particular transition state. For simplicity, we take the standard approach of MC modeling and neglect the attempt frequency effects (i.e. taking all these frequencies to be the same). We also neglect contributions to the waiting time from the transition states that were not executed. With these approximations, the average waiting time can be estimated as

$$
t_{w}=\frac{\sum_{i}^{N} \exp \left(\frac{E_{i}}{k_{B} T}\right)}{v N},
$$

where $E_{i}$ is the activation energy of the event executed in the event number $i, k_{B}$ is the Boltzmann constant, $T$ is the absolute temperature, $N$ is the total number of events (executed transition states), and $v$ is the attempt frequency. The temperature dependent effective migration energy then can be expressed as

$$
E_{E f f}(T)=k_{B} T \ln \left(v t_{w}\right) .
$$


Estimates for the interstitial atom result in $E_{E f f}(600 \mathrm{~K})=0.38 \mathrm{eV}$ and $E_{E f f}(700 \mathrm{~K})=0.37 \mathrm{eV}$ whereas for the vacancy: $E_{E f f}(900 \mathrm{~K})=1.05 \mathrm{eV}, E_{E f f}(1050 \mathrm{~K})=1.08 \mathrm{eV}$ and $E_{E f f}(1200 \mathrm{~K})=1.08 \mathrm{eV}$. These values are very close to the effective activation energies estimated from the Arrhenius treatment of tracer diffusion coefficients calculated by k-ART, shown in Fig. 4: $0.39 \mathrm{eV}$ for the interstitial atom and $1.09 \mathrm{eV}$ for the vacancy. In the cases when MD and k-ART effective activation energies are similar, the accuracy of k-ART modeling can be significantly increased by using the MD defined diffusion preexponential factors. This allows to extend accurate diffusion modeling down to temperatures not reachable by MD technique. Note that the above values are still slightly different from those estimated by $\mathrm{MD}, 0.40 \mathrm{eV}$ and $1.14 \mathrm{eV}$, highlighting the importance of the dynamic effects discussed above which might be caused by the peculiar shape of the minimum energy paths for migration described by the interatomic potentials at hand.

The interatomic potentials used here provide practically complete separation of interstitial atom and vacancy diffusion within the $\mathrm{Ni}$ and $\mathrm{Fe}$ subsystems. Although this observation is certainly subject to some uncertainties in the empirical potentials, this study provides a unique opportunity to observe how diffusion properties change within a small volume embedded into another material.

Let's consider the interstitial atom, which at the lowest simulated temperature of $600 \mathrm{~K}$ has a partial tracer diffusion coefficient ratio $D_{N i} / D_{F e} \approx 45$. Monitoring the composition of the dumbbell has shown that it contained two Ni atoms $>99 \%$ of the time. Therefore, we can conclude that the interstitial atom moves only within the Ni subsystem. It is therefore reasonable to compare its dynamical properties with that of an interstitial atom in pure Ni. The tracer diffusion coefficients presented in Fig. 3 show that the interstitial atom produces a much lower tracer diffusion coefficient in the alloy than in pure Ni. Part of this difference is related to a significant decrease of the correlation factor for interstitial atom jumps (see 
Fig. 5). The fact that the $\mathrm{Ni}$ subsystem is embedded into an Fe environment also plays a role in decreasing the diffusion rate in the equiatomic $\mathrm{NiFe}$ alloy relative to pure $\mathrm{Ni}$.

A similar phenomenon occurred for vacancy diffusion within the Fe subsystem. Although a vacancy produces mainly Fe-atom displacements, its diffusion coefficient in the alloy is much lower than that in pure Fe (see Fig. 4). As in the case of interstitial atom diffusion, a fraction of this change can be related to a decreased correlation factor (see Fig. 5) but much more should be attributed to the other embedding effects. In other words, the diffusion properties of defects in concentrated alloys are not necessarily closely related to the corresponding properties in pure metals.

The transport coefficients for interstitial and vacancy diffusion allow prediction of segregation behavior under different conditions. Under thermal aging, the alloy should suffer from standard Gibbs segregation, because vacancy sinks are depleted of Fe. This should occur because the $L_{V A C, F e}$ coefficient is negative, which means that Fe flows in the opposite direction to vacancies. Under irradiation, when interstitial atoms are produced directly, Fe depletion near interstitial sinks will be significantly enhanced by $\mathrm{Ni}$ enrichment due to interstitial diffusion with the positive $L_{I N T, N i}$.

The presented results are relevant to an equiatomic random alloy, which provides equivalent chemically homogenous contiguous regions for defect migration in each subsystem. The rate of diffusion was reduced significantly relative to the pure metals. By manipulating the alloy composition one should be able to separately affect interstitial or vacancy diffusion. By decreasing the Fe concentration below the fcc site percolation level reference, $\sim 20$ at.\%, the continuous Fe path should be broken, inhibiting vacancy diffusion. Likewise, decreasing the Ni level should inhibit interstitial diffusion. In addition, breaking both the Fe and Ni contiguous paths by adding new appropriate alloying components may damp both vacancy and interstitial diffusion, as new chemical locks are introduced along the migration pathways. 
Our observations also suggest that the evolution of microstructure in concentrated alloys under irradiation should be qualitatively different from that in dilute alloys. Concentrated alloys should damp not only the point defect diffusion studied here, but also defect cluster mobility. Several mechanisms can contribute to this play a role here, such as preferential defect formation in one of the subsystems (e.g. interstitial clusters may prefer to form in the Ni subsystem as seen in Fig. 2), or confinement of large cluster mobility to within a narrow chemically homogenous subsystem. Further study of the effects of alloy composition on both point defect and cluster migration mechanisms is in progress.

\section{Acknowledgments}

This work was supported as part of the Energy Dissipation to Defect Evolution (EDDE), an Energy Frontier Research Center funded by the U.S. Department of Energy, Office of Science, Basic Energy Sciences. LKB acknowledges additional support from a fellowship awarded by the Fonds Québécois de recherche Nature et Technologies. The authors thank Dr. A. Barashev for numerous discussions. 


\section{References}

[1] J.-W.Yeh, Journal of Materials, Alloy Design Strategies and Future Trends in High-Entropy Alloys, 65 (2013) 1759.

[2] M.-H.Tsai and J.-W.Yeh, Mater. Res. Lett., High-Entropy Alloys: A Critical Review, 2 (2014) 107.

[3] K.-Y.Tsai, M.-H.Tsai and J.-W.Yeh, Sluggish diffusion in Co-Cr-Fe-Mn-Ni high-entropy alloys, Acta Mat., 61 (2013) 4898.

[4] D.Rohrberg, K.-H.Spitzer, et al., Metall. Mater. Trans., Host Atom Diffusion in Ternary Fe-Cr-Al Alloys, 45A (2014) 269.

[5] L.S.Darken, Diffusion, mobility and their interrelation through free energy in binary metallic systems, Trans. Metall. Soc. AIME, 175 (1948) 41.

[6] J.R.Manning, Cross terms in the thermodynamic diffusion equations for multicomponent alloys, Metall. Trans., B 1 (1970) 499.

[7] A.D.Le Claire, Solute diffusion in dilute alloys, J. Nucl. Mater. 69-70 (1978) 70.

[8] A.R.Allnatt and E.L.Allnatt, Computer simulation of phenomenological coefficients for atom transport in a random alloy, Philos. Mag. A 49 (1984) 625.

[9] M.Nastar, Diffusion and coupled fluxes in concentrated alloys under irradiation: a selfconsistent mean-field approach, C. R. Physique, 9 (2008) 362-369.

[10] V.G.Vaks, A.Yu.Stroev, et al., Statistical calculations of tracer and intrinsic diffusion coefficients in concentrated alloys and estimates of microscopic parameters of diffusion from experimental data, Phylos. Mag., 95 (2015) 1536.

[11] A.V.Barashev, A.C.Arokiam, Monte Carlo modelling of $\mathrm{Cu}$ atom diffusion in $\alpha$-Fe via the vacancy mechanism, Phil. Mag. Lett. 86 (2006) 321. 
[12] C.Pareige, M.Roussel, et al., Kinetic study of phase transformation in a highly concentrated FeCr alloy: Monte Carlo simulation versus experiments, Acta Mat., 59 (2011) 2404.

[13] L.Messina, M.Nastar, et al., Exact ab initio transport coefficients in bcc $\mathrm{Fe}-X(X=\mathrm{Cr}, \mathrm{Cu}, \mathrm{Mn}, \mathrm{Ni}$, P, Si) dilute alloys, Phys. Rev., B 90 (2014) 104203.

[14] D.R.Alfonso and De Nyago Tafen, Simulation of Diffusion in FCC NiFe Binary Alloys Using Kinetic Monte Carlo Method, J. Phys. Chem., 118 (2014) 22221.

[15] J.D.Tucker, R.Najafabadi, et al., Ab initio-based diffusion theory and tracer diffusion in $\mathrm{Ni}-\mathrm{Cr}$ and Ni-Fe alloys, J. Nucl. Mater. 405 (2010) 216.

[16] L.Barnard and D.Morgan, Ab initio molecular dynamics simulation of interstitial diffusion in $\mathrm{Ni}-$ Cr alloys and implications for radiation induced segregation, J.Nucl. Mater., 449 (2014) 225.

[17] Y.N.Osetsky, S.I.Golubov and A.Serra, Study of the diffusion in binary Fe-Cu alloys, Defects and Diff. Forum, 143-147 (1997) 505.

[18] J.Marian, B.D.Wirth, et al., Dynamics of self-interstitial cluster migration in pure $\alpha$-Fe and Fe-Cu alloys, Phys.Rev., 65 (2002) 144102.

[19] A.C.Arokiam, A.V.Barashev, et al., Simulation of copper atom diffusion via the vacancy mechanism in a dilute Fe-Cu alloy, Phys. Rev. B, 71 (2005) 174205.

[20] D.Terentiev, P.Olsson, et al., On the migration and trapping of single self-interstitial atoms in dilute and concentrated $\mathrm{Fe}-\mathrm{Cr}$ alloys: Atomistic study and comparison with resistivity recovery experiments, Comp. Mat., Sci., 43 (2008) 1183.

[21] K.L.Wong, J.H.Shim and B.D.Wirth, Molecular dynamics simulations of point defect interactions in Fe-Cr alloys, J. Nucl. Mater., 367 (2007) 276.

[22] J. Marian, B.D. Wirth, et al., Cu diffusion in $\alpha$-Fe: determination of solute diffusivities using atomic-scale simulations, Comput. Mater. Sci. 31 (2004) 347. 
[23] Y.N.Osetsky, N.Anento, et al., The role of nickel in radiation damage of ferritic alloys, Acta Mat., $84(2015) 368$.

[24] X.Xu, J-P.Wang, and Y.Deng, Simultaneous analysis of three-dimensional percolation models, Front. Phys., 9 (2014) 113.

[25] N.Mousseau, L.K.Béland, et al., The Activation-Relaxation Technique: ART Nouveau and Kinetic ART. Journal of Atomic and Molecular Physics, 2012.

[26] G.Bonny, R.C.Pasianot and L.Malerba, Fe-Ni many-body potential for metallurgical applications, Modelling Simul.Mat.Sci.Eng., 17 (2009) 025010.

[27] G.Bonny, D.Terentyev, et al., Interatomic potential to study plasticity in stainless steels: the FeNiCr model alloy, Modelling Simul.Mat.Sci.Eng., 19 (2011) 085008.

[28] G.Bonny, N.Castin and D.Terentyev, Interatomic potential for studying ageing under irradiation in stainless steels: the FeNiCr model alloy, Modelling Simul.Mat.Sci.Eng., 21 (2013) 085004.

[29] S. Zhao, private communication.

[30] A.R.Allnatt, Einstein and linear response formulae for the phenomenological coefficients for isothermal matter transport in solids, J. Phys. C, 15 (1982) 5605.

[31] Y.N.Osetsky, Atomistic Study of Diffusional Mass Transport in Metals, Defect and Diff. Forum, 188-190 (2001) 71.

[32] N.Anento, A.Serra and Y.N.Osetsky, Atomistic study of multimechanism diffusion by selfinterstitial defects in $\alpha$-Fe, Modelling Simul.Mat.Sci.Eng., 18 (2010) 025008.

[33] X.Haixuan, Y.N.Osetsky and R.E.Stoller, Self-evolving atomistic kinetic Monte Carlo: fundamentals and applications J. Phys.: Condens. Matter, 24 (2012) 375402. 


\section{Appendix}

A random equiatomic $\mathrm{NiFe}$ alloy was created and relaxed at zero pressure. A vacancy or interstitial atom $(<100>$ dumbbell) was introduced before a second relaxation. Interstitial dumbbells were created by an appropriate shift of a given $\mathrm{Ni}$ or $\mathrm{Fe}$ atom off its lattice site while inserting either an atom of the same or different type. This allowed us to cover all possible dumbbell combinations such as Ni-Ni, Fe-Fe and Ni-Fe. The modeling system is an fcc cubic lattice oriented along the $<100>$ directions with a side length of $10 \mathrm{a}$ ( $\mathrm{a}$ is the lattice parameter) and containing 4000 lattice sites. Equation of motion were integrated with a constant time step equal to 2 fs at $\mathrm{T}<900 \mathrm{~K}$ and 1 fs at $\mathrm{T} \geq 900 \mathrm{~K}$. A large number of defect jumps, i.e. a long trajectory, is necessary to obtain a statistically significant treatment of diffusion data. A few thousand jumps for vacancies and up to $4 \times 10^{5}$ jumps for interstitial atoms were generated. Depending on the defect migration energy and diffusion mechanism, a time up to $\sim 1 \mu$ s was simulated. The total time for all the defects and temperatures reached more than $14 \mu \mathrm{s}$. This time is significantly longer than that simulated so far in MD diffusion studies [17-23]. However even this was not enough to obtain accurate results for vacancies in $\mathrm{Ni}$ and $\mathrm{Ni}-\mathrm{Fe}$ at low temperature. An example of a long but unsatisfactory simulation is presented in Fig. A1. The atomic squared displacements (ASDs) associated with a single vacancy as a function of time is plotted; the insets indicate the number of jumps observed in each case. The total number of vacancy jumps in $\mathrm{Ni}(150)$ and alloy (375) and the corresponding increase in ASD are too small to estimate the tracer diffusion coefficient accurately. (For example, the convergence of transport coefficients was achieved when $\sim 100$ of 40-jumps segments was treated in the alloy (see Fig.6)).

Interstitial atom and vacancy diffusion at low temperatures was also modeled using the kinetic activation-relaxation technique (k-ART). This technique is an extension of adaptive kinetic Monte Carlo (kMC) and allows modeling slow dynamic processes at the atomic scale. In k-ART, the activation- 
relaxation technique nouveau (ARTn) [25] is used to find the spectrum of first-order saddle points on the potential energy surface connected to a given defect configuration, as well as the stable states they lead to. This spectrum then is used as in conventional kMC to chose an activated event to advance in time and determine the trajectory of the defect. The whole system is then relaxed and ARTn is applied again to find new transition states (if the new configuration was previously visited, k-ART will also use events from its catalog). A powerful feature of k-ART is that it considers a large number of realistic transition states, which can be interpreted as defect diffusion mechanisms. For each KMC step we performed 4 searches centered on each of the 12 neighbors of a vacancy or of an interstitial (as well as on the two interstitial atoms). While we cannot claim with certainty that we have an "complete" catalog, it is certainly substantial. The main disadvantage of the current implementation of k-ART is that it uses a constant pre-exponential factor $(10 \mathrm{THz})$. In the case of simple defects this constant can be fitted directly to MD data (see e.g. [34]). Because of the great variety of possible transition states such a fitting in concentrated alloys may demands quite long simulations. In the case when MD and k-ART effective activation energies are similar, the MD defined diffusion pre-exponential factors can be used in k-ART calculations. The opposite case indicates that strong dynamics effect modelled by MD are not described correctly by harmonic/quasiharmonic transition state theory in the basis of modern on-the-fly kMC techniques. Nevertheless k-ART and similar on-the-fly kMC techniques are among a small number of methods able to model kinetic processes governed by large activation energies at low temperatures.

MD and k-ART produce atomic-level output that can be used to treat diffusion and mass transport in detail. A general approach is described in [25]. The description of diffusion and mass transport is based on the generalized Einstein equations for transport, or phenomenological, coefficients $L_{i j}[8,30]$ :

$$
L_{i j}=\frac{\left\langle\boldsymbol{R}_{i} \boldsymbol{R}_{j}\right\rangle}{6 t}
$$


where $i$ and $j$ are considered objects, e.g. defects or atoms of a certain type, $\boldsymbol{R}_{i}$ is the total displacement vector of object $i$ over time $t$ and the brackets denote the average over the entire defect ensemble. The matrix of transport coefficients contains information about defect diffusivity and atomic transport. For example if $i=j$ is a defect (here vacancy or interstitial atom) or atoms of a particular alloying component, then $L_{i j}$ is either $D_{d e f}-$ the defect diffusion coefficient, or $D^{*}{ }_{i}-$ the partial tracer diffusion coefficient of the component $i$. If $i$ is a defect, e.g. vacancy, and $j$ denotes atoms of a particular alloy component, e.g. Ni, then $L_{V A C, N i}$ describes where and how fast vacancies transport $\mathrm{Ni}$ atoms. In practice atomic-scale modeling generates only one or a few long defect trajectories. Decomposing a defect migration trajectory into segments is used to improve statistical convergence. In the trajectory decomposition method, the most important issue is the segment length; it must be chosen to include all the defect jump correlations. Typically, the diffusion coefficient converges when segments include a few tens of jumps (e.g. see Fig. 6). Of course, the number of these segments should be large enough to obtain a small error of the mean value of the diffusion coefficients $[31,32]$.

Defect diffusion coefficients can also be estimated using an atomistic theory of diffusion, by treating defect jump frequencies and dynamic correlation factor, $f_{c}$ (see e.g. [31]). Correlation factors for defect jumps are defined as $f_{c}=(1+\langle\cos (\Theta)\rangle /(1-\langle\cos (\Theta)\rangle)$, where $\Theta$ is the angle between the consecutive defect jump vectors. The dynamic correlation factor should not to be confused with the tracer correlation factor that defines efficiency of atomic transport for the random walk defect diffusion (see e.g. [31]). Averaged over a large number of jumps, correlation factors reflect how efficiently jumps contribute to the defect spatial trajectories. The most crucial element is the total number of successful jumps treated. For vacancy diffusion, where $f_{c}$ is typically large, a few thousand jumps are usually enough. However, diffusion of interstitial atoms especially in concentrated alloys is usually strongly correlated and about an 
order of magnitude more jumps need to be treated to accurately estimate the correlation factor and defect diffusion coefficient. 


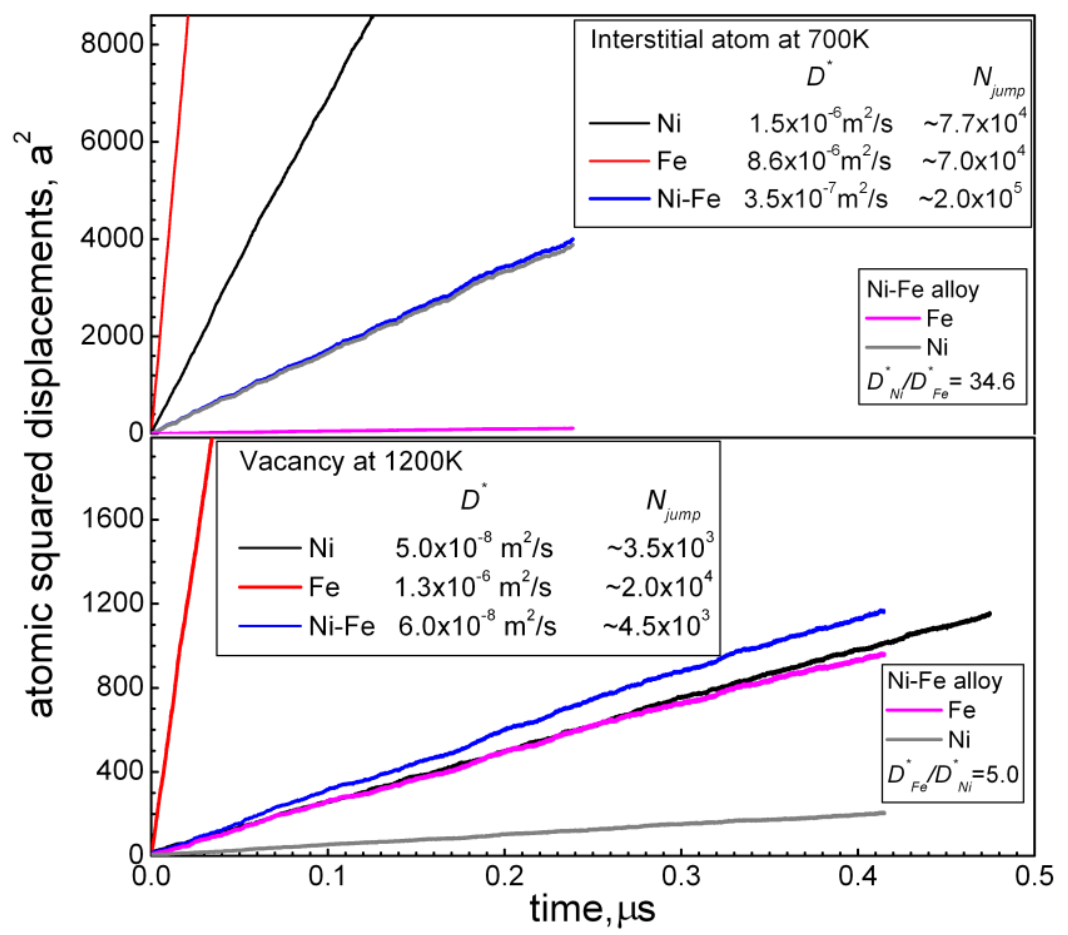

Fig.1. Atomic square displacements as function of time obtained in MD modeling of an interstitial atom (upper plot) and vacancy (lower plot) in pure $\mathrm{Ni}, \mathrm{Fe}$ and $\mathrm{Ni}-\mathrm{Fe}$ alloy. Ni-atom displacements (grey line) and total ASD in Ni-Fe alloy (blue line) are practically overlapped on the upper plot indicating a very small contribution from Fe-atom displacements in the interstitial diffusion mechanism (pink line). The insets indicate tracer diffusion coefficients obtained by linear fitting of the atomic square displacements $\left(D^{*}\right)$, number of defect jumps observed $\left(N_{\text {jump }}\right)$ and the ratio of partial diffusion coefficients in the alloy. 


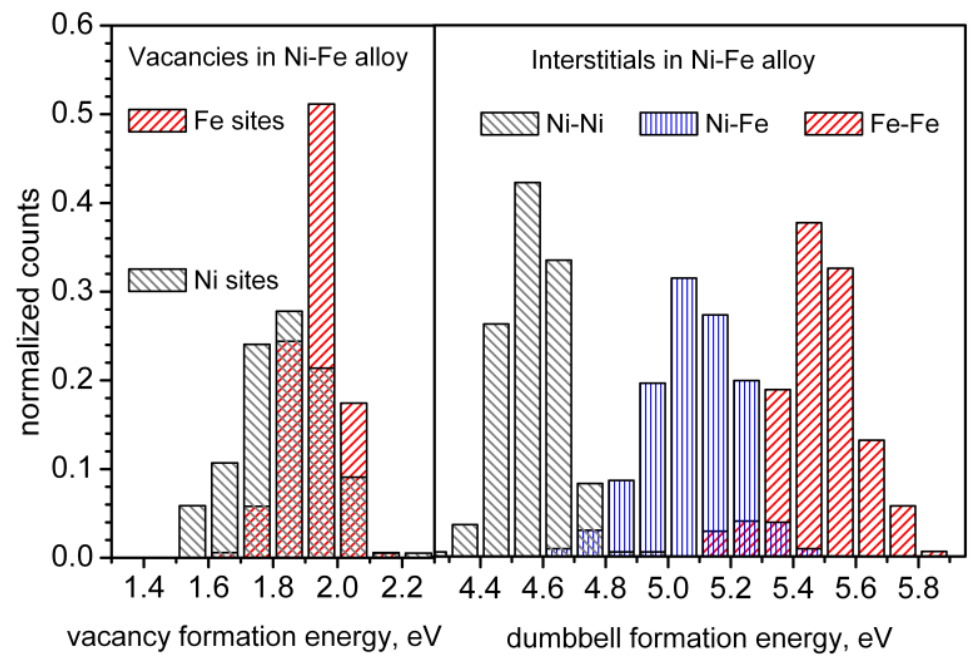

Fig.2. Distribution of formation energies for a vacancy in different atomic sites and interstitial atoms of different composition obtained in the Ni-Fe alloy. About 3000 sites in the equiatomic NiFe random alloy were tested in each case.

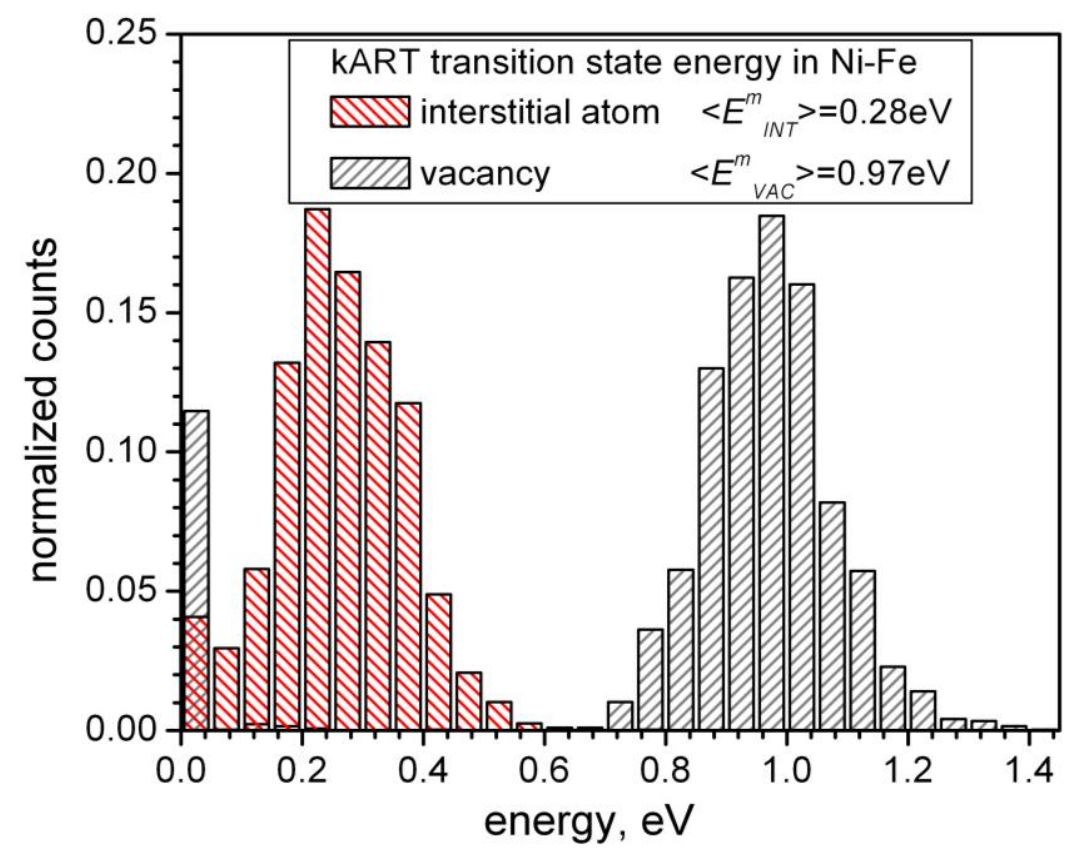

Fig.3. Barriers executed during k-ART modeling of vacancy diffusion at $1200 \mathrm{~K}$ and interstitial atom diffusion at $600 \mathrm{~K}$ in Ni-Fe alloy. More than 3000 transition states were tested for each defect. Note that a few of very low energy barriers for the both defects are related to metastable configurations near the halfway points between some stable defect configurations. 


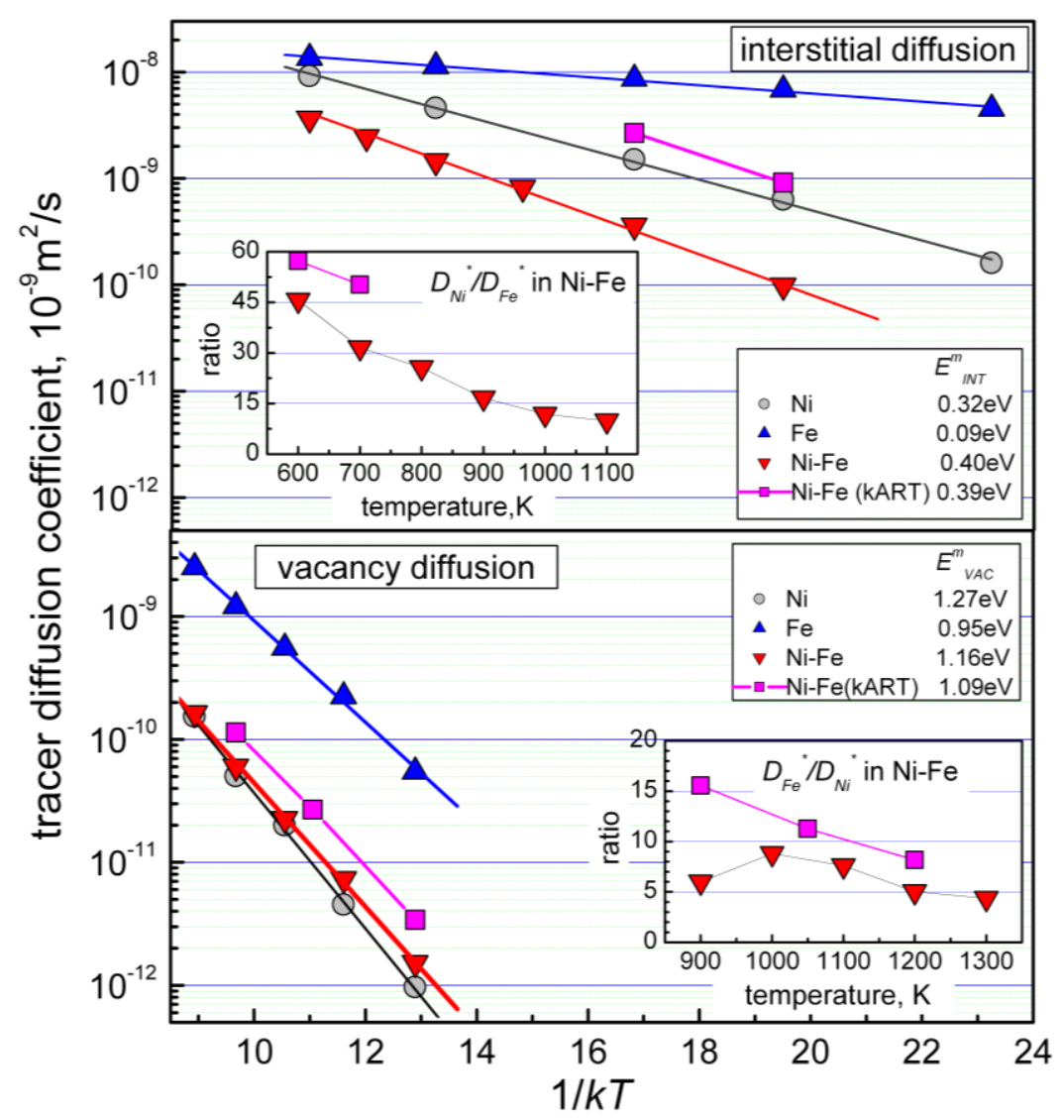

Fig.4. Tracer diffusion coefficients due to interstitial atom (upper plot) and vacancy (lower plot) diffusion. Blue - pure Fe, gray - pure Ni, red - Ni-Fe alloy, pink - k-ART results for Fe-Ni alloy. The inserts show the temperature dependence of the $D_{N i} / D_{F e}$ ratio for interstitial (upper plot) and $D_{F e} / D_{N i}$ ratio for vacancy (lower plot) diffusion in Ni-Fe alloy. 


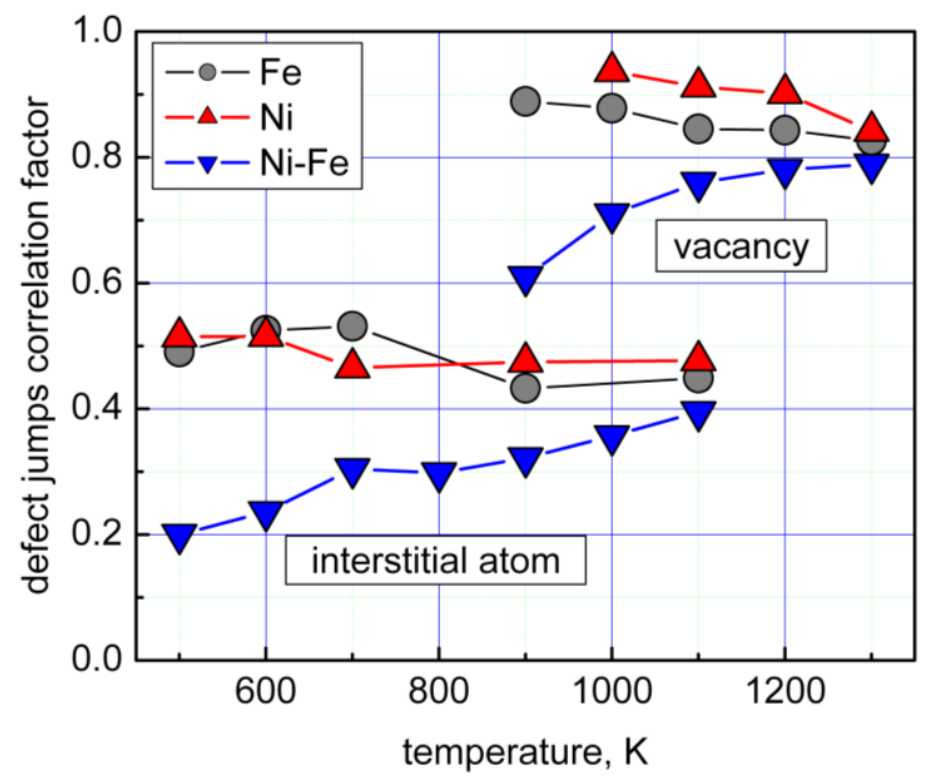

Fig.5. Correlation factor of vacancy and interstitial atom jumps in pure metals and the Ni-Fe alloy.

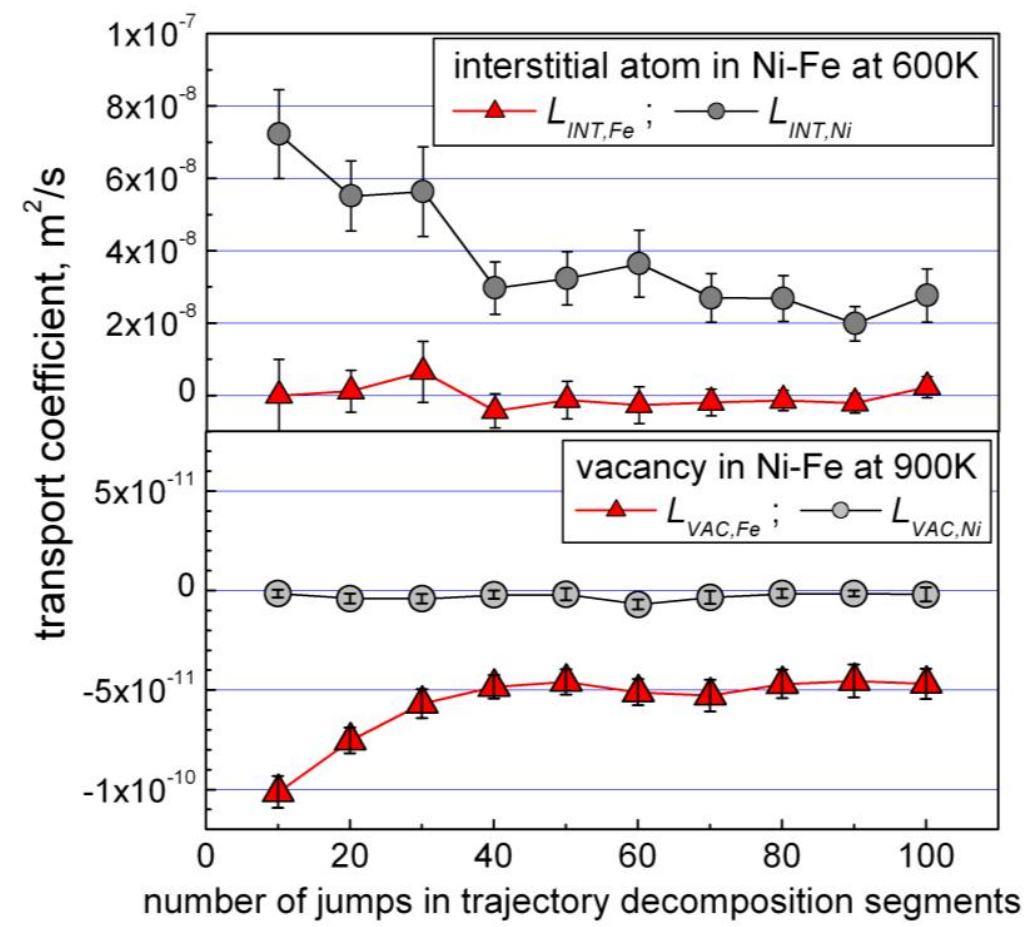

Fig.6. Convergence of transport coefficients in Ni-Fe alloy treated using the trajectory decomposition technique of interstitial atom and vacancy diffusion. 


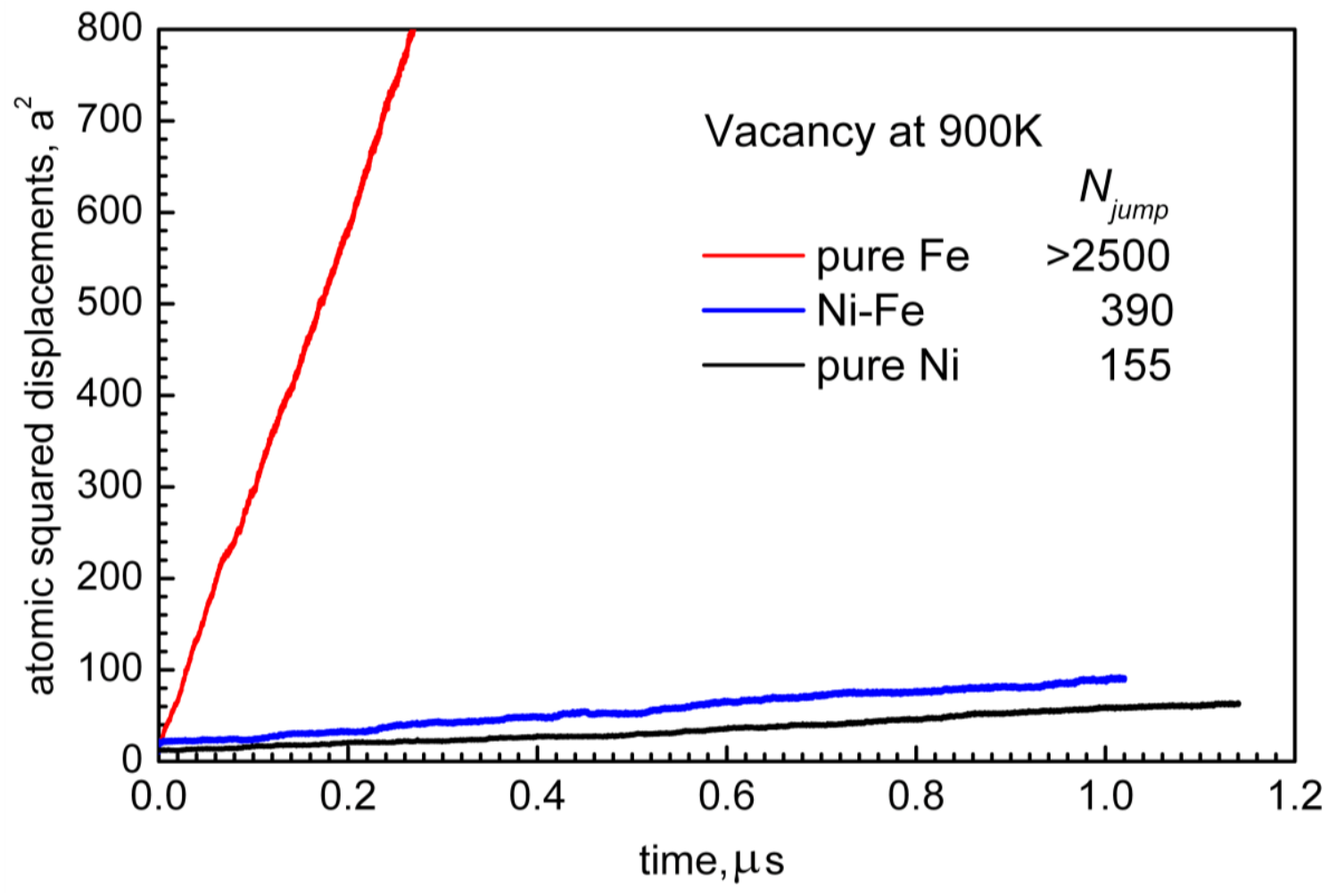

Fig. A1. Examples of atomic square displacements (ASD) due to vacancy migration at $900 \mathrm{~K}$ in pure metals and the NiFe alloy. The number of vacancy jumps observed is indicated in the legend. 
Table 1. Properties of point defects in eV calculated with different Ni and Fe potentials. Static data, calculated at $\mathrm{T}=0 \mathrm{~K}$ indicated at the top, $\mathrm{DFT}$ results are indicated in parenthesis and current work MD results for activation energy from tracer diffusion coefficient are at the bottom.

\begin{tabular}{|c|c|c|c|c|}
\hline \multirow{2}{*}{ Me/IAP } & \multicolumn{2}{|c|}{ vacancy } & \multicolumn{2}{c|}{ self interstitial atom } \\
\cline { 2 - 5 } & $E^{f}$ & $E^{m}$ & $E^{f}$ & $E^{m}$ \\
\hline \multirow{2}{*}{$\mathrm{Ni}$} & 1.48 & 1.17 & 4.08 & 0.33 \\
$\mathrm{~B} 2011$ & $(1.37-1.79)$ & $(1.08-1.30)$ & $(4.07-4.11)$ & $(0.14)$ \\
& - & 1.32 & - & 0.32 \\
\hline \multirow{2}{*}{$\mathrm{Fe}$} & 1.94 & 0.61 & 3.60 & 0.38 \\
$\mathrm{~B} 2011$ & $(1.82-1.95)$ & $(0.62)$ & $(3.20-3.62)$ & - \\
& - & 0.92 & - & 0.09 \\
\hline \multirow{2}{*}{$\mathrm{Ni}$} & 1.39 & 1.09 & 5.83 & 0.18 \\
$\mathrm{~B} 2013$ & $(1.37-1.79)$ & $(1.08-1.30)$ & $(4.07-4.11)$ & $(0.14)$ \\
& - & 1.14 & - & 0.16 \\
\hline \multirow{2}{*}{$\mathrm{Fe}$} & 1.87 & 0.64 & 5.25 & 0.25 \\
$\mathrm{~B} 2013$ & $(1.82-1.95)$ & $(0.62)$ & $(3.20-3.62)$ & - \\
& - & 0.98 & - & 0.30 \\
\hline
\end{tabular}


Tracer diffusion coefficients of point defect estimated in $\mathrm{Ni}$-Fe equiatomic alloy by molecular dynamic (MD) and kinetic activation-relaxation technique (k-ART)

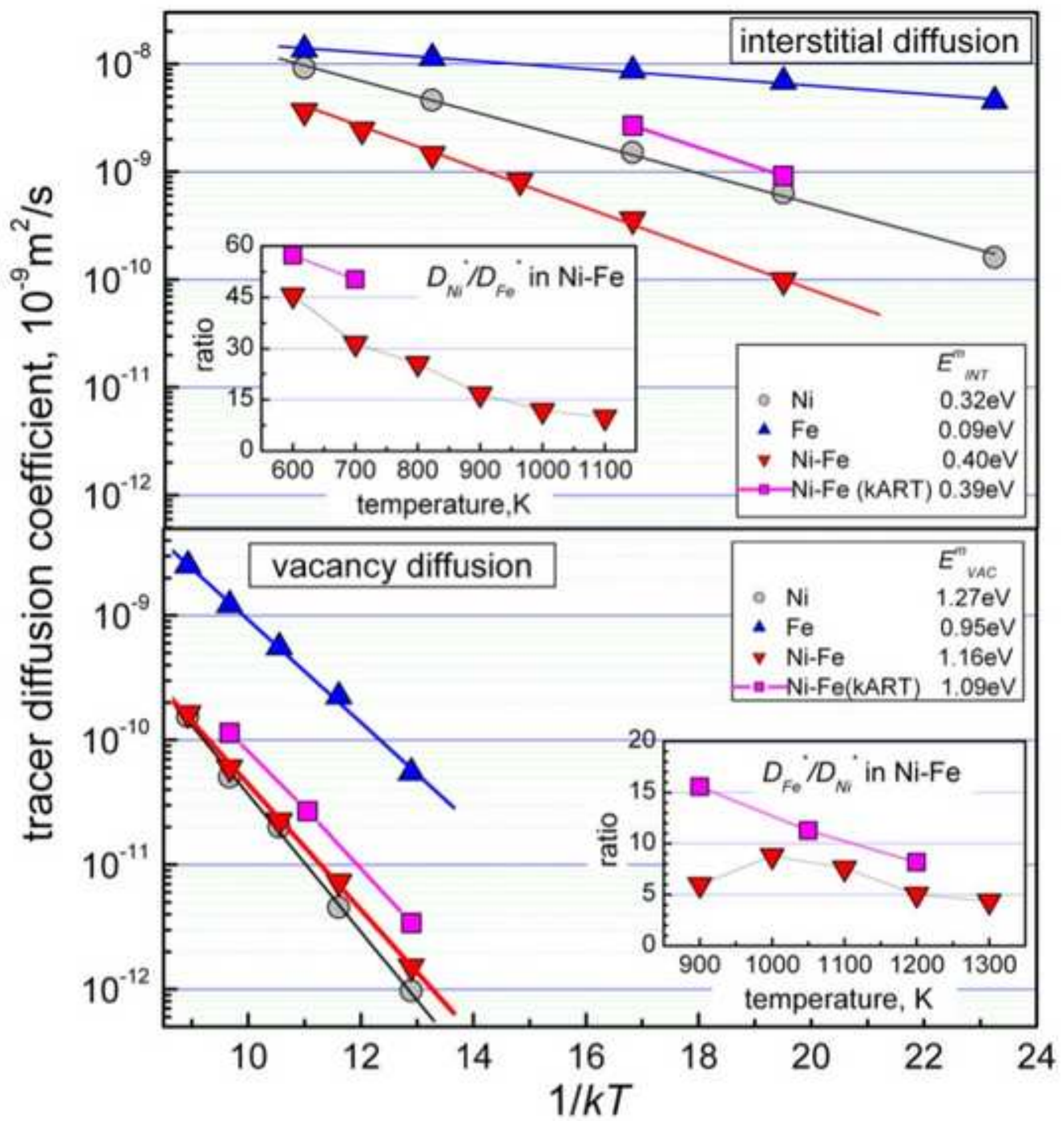

